\title{
The Covid-19 Outbreak and Pediatric Neurosurgery guidelines
}

\author{
Ricardo Santos de Oliveira, MD, PhD ${ }^{1 *}$ - Matheus Fernando Manzolli Ballestero, MD, MSc ${ }^{2 \star}$ \\ Received: 26 March 2020 / Accepted: 26 March 2020
}

The latest threat to global health is the ongoing outbreak of the respiratory disease that was recently given the name Coronavirus Disease 2019 (Covid-19). Covid-19 was recognized in December 2019 [1]. It was rapidly shown to be caused by a novel coronavirus that is structurally related to the virus that causes severe acute respiratory syndrome (SARS). As in two preceding instances of emergence of coronavirus disease in the past 18 years [2] - SARS (2002 and 2003) and Middle East respiratory syndrome (MERS) (2012 to the present) — the Covid-19 outbreak has posed critical challenges for the public health, research, and medical communities.

The Brazilian Society for Pediatric Neurosurgery (SBNPed) announced that all elective surgeries, nonessential pediatric neurosurgeries be delayed during the 2020 Novel Coronavirus (COVID-19) outbreak (figure 1).

The recommendations provide a framework for hospitals and clinicians to implement immediately during the COVID-19 response, as more healthcare providers are increasingly being asked to assist with the COVID-19 response, it is critical that they consider whether non-essential surgeries and procedures can be delayed.

The recommendations outline factors that should be considered for postponing elective surgeries. Those factors include patient risk factors, availability of beds, ventilators, staff and personal protective equipment (PPE), and the urgency of the procedure. This will help providers to focus on addressing more urgent cases and preserve resources needed for the

'Division of Neurosurgery, Department of Surgery and Anatomy, University Hospital, Ribeirão Preto Medical School, University of São Paulo, Ribeirão Preto, Brazil

${ }^{2}$ Department of Medicine, Federal University of São Carlos, São Carlos, Brazil

* Equal contribution to the manuscript

To whom correspondence should be addressed: Ricardo Santos de Oliveira, MD, PhD [E-mail: rsoliveira30@gmail.com]
COVID-19 response. The decision about proceeding with non-essential surgeries and procedures will be made at the local level by the clinician, patient, parents, hospital, and state and local health departments.

To ensure planning strategies to carry out urgent/emergency operations during the pandemic. Rational: 1) to ensure an adequate treatment for patients, those with trauma, hemorrhage or severe infection, for example, who require immediate surgery; 2) to prepare and guide all the healthcare professionals on how to act, adequately protecting themselves with PPE; 3) to allow the minimum required people in the surgical room, thus preventing the risk of contamination of other individuals. In this regard, this is a mandatory perception of quality and safety initiatives not only for disasters and pandemics.

Some overarching principles for all cases include the following:

1. Be aware that while some of the following triaging guidelines include a "Level 1" (e.g., lowest level of COVID-19 acuity) in the recommendations, one must be aware that the rates of COVID-19 are predicted to skyrocket in this year, and the overarching recommendation is to prepare for markedly increased rates when triaging elective cases at present.

2. Patients should receive appropriate and timely surgical care, including operative management, based on sound surgical judgment and availability of resources.

3. Consider nonoperative management whenever it is clinically appropriate for the patient.

4. Consider waiting on results of COVID-19 testing in patients who may be infected.

5. Avoid emergency surgical procedures at night when possible due to limited team staffing.

6. Aerosol generating procedures (AGPs) increase risk to the health care worker but may not be avoidable. For patients who are or may be infected, AGPs should only be performed while wearing full PPE including an N95 mask or powered, air-purifying respirator (PAPR) that has been designed for the OR. Examples of known and possible AGPs include: intubation, tracheostomy. 
7. Endonasal procedures should be delayed because of high risk to be contaminate [3]

8. Covid-19 laboratorial tests should be done in patients with signs and symptoms that need to be operate.

The reality is clear and the stakes are high: we need to preserve personal protective equipment for those on the front lines of this fight.

Key words: Pediatric neurosurgery, Neurosurgery, COVID-19, Health care

\section{References}

1. World Health Organization. Pneumonia of unknown cause-China. Emergencies preparedness, response, Disease outbreak news, World Health Organization (WHO). 2020 Jan.

2. de Wit E, van Doremalen N, Falzarano D, Munster VJ. SARS and MERS: recent insights into emerging coronaviruses. Nature Reviews Microbiology. 2016 Aug;14(8):523.

3. Patel Z M, Fernandez-Miranda J, Hwang PH, Nayak JV, Dodd R, Sajjadi H, Jackler RK. Precautions for endoscopic transnasal skull base surgery during the Covid-19. Neurosurgery. In press

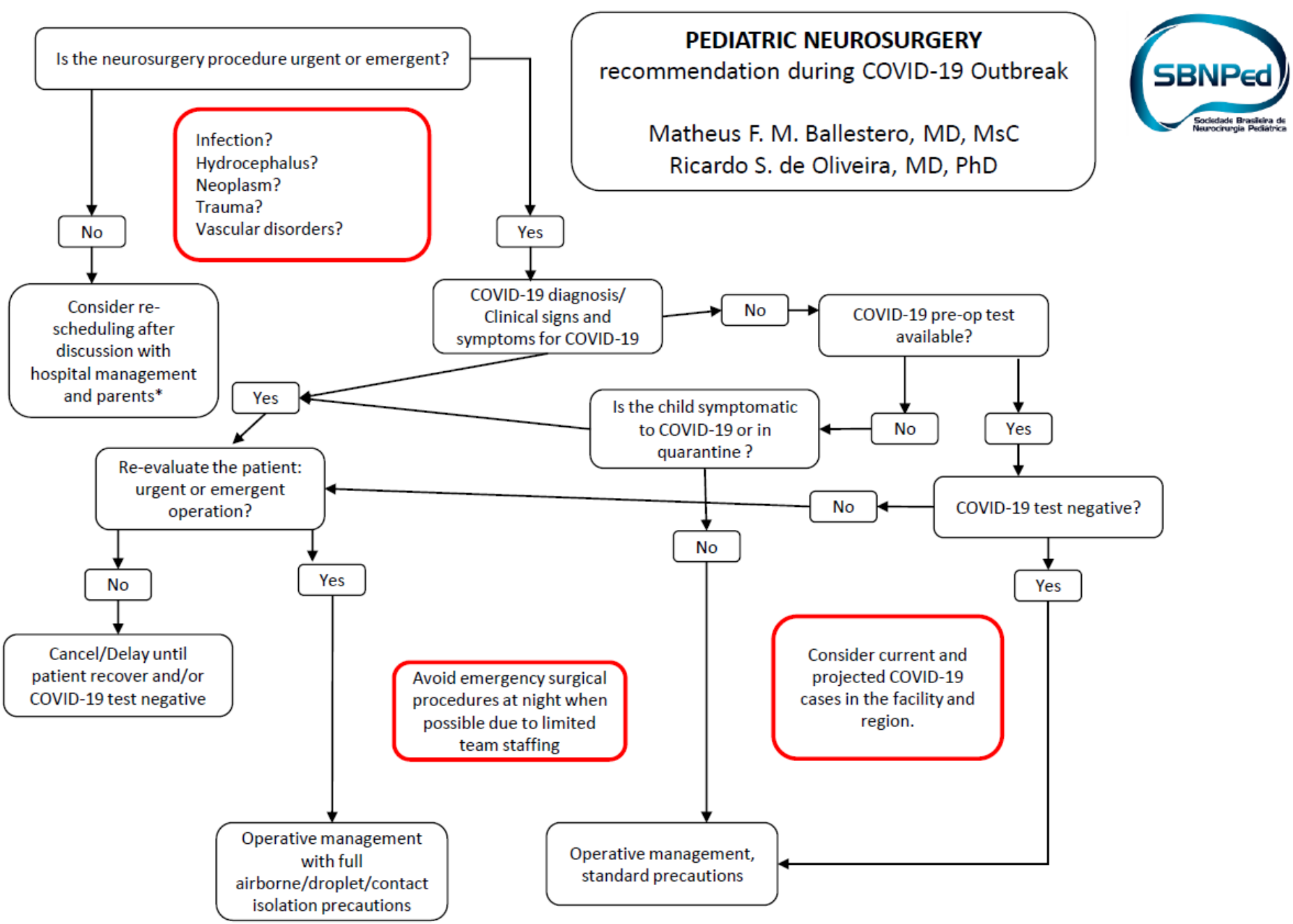

* Elective surgeries and non-essential medical and surgical procedures should be delayed until the COVID-19 outbreak ends. Consider a range of factors when deciding whether to postpone a surgery or procedure, including patient risk factors, bed availability, staffing and personal protective equipment supplies

Figure 1 - Brazilian Society of Pediatric Neurosurgery recommendation during COVID-19 Outbrake 